\title{
Evaluation of an acoustic Doppler technique for bed-load transport measurements in sand-bed Rivers
}

\author{
$S$. Conevski ${ }^{1,2}, A$. Winterscheid ${ }^{3}, N$. Ruther ${ }^{1}, M$. Guerrero ${ }^{2}, C$. Rennie ${ }^{4}$ \\ ${ }^{1}$ Department of Civil and Environmental Engineering, Norwegian University of Science and \\ Technology, Trondheim, Norway. slaven.conevski@ntnu.no; nils.ruther@ntnu.no \\ ${ }^{2}$ Department of Civil, Chemical, Environmental, and Materials Engineering, University of Bologna, \\ Italy.massimo.guerrero@unibo.it; slaven.conevski2@unibo.it \\ ${ }^{3}$ Federal Institute of Hydrology, Department Groundwater, Geology, River Morphology, Koblenz, \\ Germany winterscheid@bafg.de \\ ${ }^{4}$ Department of Civil Engineering, University of Ottawa, Ottawa, Canada. crennie@genie.uOttawa.ca
}

\begin{abstract}
The bottom tracking (BT) feature of the acoustic Doppler current profilers (ADCP) have emerged as a promising technique in evaluating the bed load. Strong statistical correlations are reported between the ADCP BT velocity and the transport rate obtained by physical sampling or dune tracking; however, these relations are strictly site-specific and a local calibration is necessary. The direct physical sampling is very labor intensive and it is prone to high instrument uncertainty. The aim of this work is to develop a methodology for evaluating the bed load transport using commercial ADCPs without calibration with physical samples. Relatively long stationary measurements were performed in a sand-bed and sand gravel rivers, using three different $\mathrm{ADCPs}$ working at $3 \mathrm{MHz}, 1.2 \mathrm{MHz}$ and $0.6 \mathrm{MHz}$. Simultaneously, bed load samples were collected with physical samplers, and the riverbed was closely observed with digital cameras mounted on the samplers. It is demonstrated that the kinematic transport model can yield a relatively good estimate of the transport rate by directly using filtered apparent velocity, the knowledge of the hydraulic conditions and instrument-related calibration coefficients. Additionally, the ADCP data can help in qualitative assessment of the physical sampling. Future investigation of the backscattering echo and further confirmation of the BT apparent velocity should be performed in laboratory-controlled conditions.
\end{abstract}

\section{Introduction}

The quantification of the bedload transport in fluvial environments is a notoriously difficult and labor-intensive task. The temporal and spatial variability of the bed load together with the instrument disturbance can induce stochastic and systematic uncertainties. These data are usually considered as unreliable, which complicates the further elaboration of the sediment transport behavior. Statistically valid measurements are extremely important for the evaluation of the sediment transport masses, especially in large navigable or heavily exploited rivers. Recently, many studies showed that the measurements with the bottom tracking (BT) feature of the acoustic Doppler current profilers (ADCP) have emerged as a promising technique in evaluating the bed load $[1,2,3,4]$. The use of these non-intrusive techniques could significantly reduce the uncertainty. Firstly, they do not disturb the riverbed and facilitate continuous measurements of the bed load. Secondly, they are easy to deploy and more frequent measurements can be expected. Widespread implementation of the ADCP BT bedload technique would reduce the scarcity and statistical uncertainty of bed load data. Strong statistical correlations are reported between the ADCP BT velocity and the transport rate obtained by direct physical sampling or dune tracking [5]; however, these relations are strictly site-specific and a local calibration is necessary. Previous laboratory research has demonstrated that with rather simple filtering and smoothing of the ADCP BT velocity (denoted as the apparent velocity. $v_{a}$ ), one can obtain relatively correct spatially averaged 
velocity of the bed surface [6]. Deviation in the results is assigned to the coupling between acoustic waves at the given instrumental frequency, the size distribution of mobile particles (i.e., PSD) and the riverbed morphology.

The aim of this work is to develop a methodology for evaluating the bed load transport using commercial ADCPs without certain calibration to the physical samples. However, it is necessary to know the hydraulic conditions and the particle size distribution (PSD) of the riverbed material that could be taken from some previous studies. The necessary hydraulic conditions can be obtained from the ADCPs water velocity column and used for further analysis of the bedload transport. The main goal of this study is to assess the capability of the ADCP measurements to over come the already existing problems with the bedload samplers and help the final estimation of the bedload mases.

\subsection{Study sites}

The measurement campaign in Germany took place in two cross sections of the lower Oder River along the German-Polish border $\left(52.8743^{\circ} \mathrm{N}, 14.1419^{\circ} \mathrm{E}\right)$. Seven positions were chosen in the one cross section and eight positions in the other cross section. The positions were chosen trying to avoid the groynes influence. The average discharge was $800 \mathrm{~m}^{3} / \mathrm{s}$, with a maximum average velocity in the middle of the river of $1 \mathrm{~m} / \mathrm{s}$. The total width of the crosssection was $200-300 \mathrm{~m}$ including the groynes. The water depth varied from $2.5 \mathrm{~m}$ near the banks to $5.2 \mathrm{~m}$ in the thalweg. The annual mean discharge is around $500 \mathrm{~m}^{3} / \mathrm{s}$ and the mean annual maximum discharge is around $1200 \mathrm{~m}^{3 / \mathrm{s}}$ (http://geoportal.bafg.de ); thus the measured flow had elevated discharge but was not a flood event. From the morphological perspective the Oder River is a typically sandy river with well-characterized slow dune migration. The D50 (median diameter) of the bedload in the measurement reach was $0.75 \mathrm{~mm}$ with standard deviation $(s t d=D 84 / D 16)$ of $1.5 \mathrm{~mm}$. The particle size distribution is relatively homogeneous through the entire cross section.

The measurements in Albania were performed in the sand-gravel reach of the small river Tomorrica $\left(40.8271^{\circ} \mathrm{N}, 20.18639^{\circ} \mathrm{E}\right)$ where the average velocity in the sampling area of the ADCP reached $1.1 \mathrm{~m} / \mathrm{s}$. The Tomorrica is a tributary of the Devoll River flowing from the Tomorri peak to the $1-\mathrm{km}$-wide alluvial valley of the Devoll with typical braid morphology. In total 25 measurement positions are included in this study from two campaigns obtained in December 2016 and March 2017. The discharge varied between 2.5$3.5 \mathrm{~m}^{3} / \mathrm{s}$, and the measuring cross section width around $9 \mathrm{~m}$ with variable depths at maximum of $0.6 \mathrm{~m}$. The sand-gravel bed has $D 50$ that varied between $0.5-4 \mathrm{~mm}$ with considerably higher std of 4-6 mm. The catchment is characterized with high coefficient of variation of the discharge, thus the sediment transport occurs very randomly, consisting of rocks, cobbles and gravel during the floods and sand during the annual flow discharges. There were fully armored parts of the river with some sand passing over and sand-gravel mixed areas. The measurements were conducted in the sandy parts with $0.45-0.7 \mathrm{~m}$ average depth of the measurement area.

It has to be mentioned that the PSD distribution analyses were done only on the mobile bed material that was actually sampled during the measurements, therefore the general riverbed characteristics should not be identified with these PSDs. The main goal is to estimate their acoustical footprint of the particles that are forming the active bedload layer.

\subsection{ADCP operation}

In total four different ADCPs were used in the campaigns. Two Teledyne RDI ADCPs (RioGrande $1.2 \mathrm{MHz}$ and $0.6 \mathrm{MHz}$, four beam configuration) and one SonTek(M9 $3 \mathrm{MHz}-$ working with four slanted-beam Janus configuration and one vertical beam of $0.5 \mathrm{MHz}$ ) were used in Germany. In Albania, only one RDI (RiverPro $1.2 \mathrm{MHz}$, with four slanted-beam configuration plus additional vertical beam) was used. The ADCP-type instruments are designed to measure the water velocities at several ranges to determine discharge and to give 
information about the flow field. These monostatic sonars have usually four beams, i.e., four transducers that emit acoustic pulses and receive back the backscattered echoes intensities $(B s E I)$. The signal is scattered from the particles that enter the acoustic beams and from the acoustical impermeable boundary, namely the immobile riverbed. The returned echoes contain information from the acoustical properties of each object they are reflected from and a shift of frequency (e.g. Doppler shift) to the object velocity, thus the object velocity in the direction of the beam can be easily calculated and then transformed in the coordinate system of interest, which is well explained by Rennie et. al [1]. The ADCPs have a separate signal called the bottom tracking (BT) mode to identify the bottom and its position. It uses a longer pulse and in case of mobile riverbed it is significantly biased. The bias(e.g. $\left.v_{a}=v_{g p s}-v_{B T}\right)$ can be exploited to calculate the average velocity of the river bed surface, denoted as the apparent velocity. The apparent velocity in case of fixed stationary measurements is equal to the BT velocity $\left(v_{a}=v_{B T}\right)$, since the GPS velocity $\left(v_{g p s}\right)$ is zero. Nevertheless, the received echo is not only scattered from the mobile bedload, but it may be also influenced by the suspended particles close to riverbed, the immobile particles (immobile rough surface) and the riverbed morphology. Considering the complicated transmission of the signal in the active layer of the bedload and the scattering from the highly porous immobile and deformed riverbed the returned signal is noisy and contains erroneous data [7]. The erroneous data in the bedload velocity is more common for the areas with scarce transport. It is also instrument related how each signal will behave in the complex two-phase insonified acoustic volume. For example, the working frequency $(f)$, the pulse length, the grazing angle and the instrument specific coefficients can significantly change the results. The returned signal contains backscattered information of the immobile particles (rough surface scattering) and the mobile particles (volume scattering). For the acoustic simplicity only the particles in motion are considered as the active layer of the riverbed, the vibrating and potentially mobile are considered as the part of the rough surface scattering, thus the immobile particles.

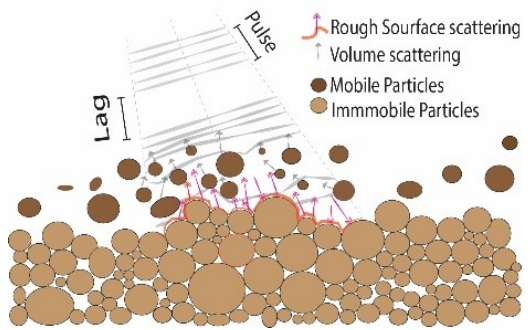

Fig. 1. Acoustic footprint of the riverbed from one ADCP beam and the sonar equation

$$
B S E I=S L+S V+K-2 T S-20 \log R,
$$

where the $S L$ is the emitted source level, $S V$ is the backscattering strength, $K$ is the instrument related coefficient, $T S$ are the transmission losses in the water column and in the active layer of the riverbed. The last component is related with the spreading of the signal at different water depth $(R)$. The $S V$ and $T S$ are varying in different conditions of bedload transport, different PSD and different instrument frequency $(f)$ and other instrument related parameters. SV and TS are depending differently from the two types of scattering mentioned above. The variability of the Doppler velocity calculation is strongly related with the variability of the $B S E I$, hence it is used into the calculation of the Doppler velocity. The correct interpretation of the components of the returned signal from the riverbed (e.g. $S V$ and $T S$ ) would lead to a better understanding of the velocity information and it will yield additional information about the thickness of the active layer.

A possible high concentration of suspended sand above the bed can bias the results showing the velocity of the suspended particles moving in the water layer above the bed and not the bedload. It is called the water bias [1] and has to be checked before the analysis. In both campaigns the velocity of the last cell of the water velocity profile is significantly higher than 
the apparent velocity, thus the water bias is not an issue in this study. It is worth nothing that the scattering from the riverbed material is expected to have significantly higher BS strength than the fine suspended sand or silt that would eventually induce the bias.

\subsection{Physical Sampling}

Three physical samplers were collected on each position at almost same time interval with the ADCPs. In Tommorice river, small Helley-Smith (H-S) sampler (width of the inlet $b=7.65 \mathrm{~cm}$ ) was used and in Germany two custom-made samplers were used [8]. The width of each was $b=16 \mathrm{~cm}$ but they had different mesh sizes $(0.5 \mathrm{~mm}$ and $1.4 \mathrm{~mm})$ of the collecting bag resulting with slightly different collection efficiency. Physical samplers are disturbing the original hydraulic conditions once they enter the river, and require hard manual labor and organization effort. There is a wide range of uncertainty. First of all the human operation and experience, but the pulsating behavior of the bedload transport that can be caused by the instantaneous variations in movement [9]. Additionally, the uncertainty can be induced by some systematic problems, such as improper sampler design, that may cause clogging of the collecting bag and prevents the entry of bed-load, or the loss of material when lifting up the sampler after each finish of the measurement. The custom-made bedload samplers demonstrate constant uncertainty during sampling in the sandy rivers. Due to the specific design the typically underestimated the transport rates. The most sensitive part was the mash size of the sampler's bag, where the coarser one loses material while the other one is prone to clogging. The mounted cameras on the samplers (only in Germany) frame also helped to clearly identify the problems of clogging, loosing material, debris obstacles, rocks blocking the nozzle, etc. The standard H-S sampler used in Albania is very sensitive to a correct positioning in the river. The samplers were not at exactly the same position as the ADCPs, therefore some deviation is expected due to the spatial and temporal natural variability of the river morphology and the transport processes.

\section{Methodology}

\subsection{ADCP data analysis}

The collected data from the acoustic instruments cannot be used directly and there is noncommercial software that acquires instant and accurate estimation of the BT velocity. The ADCP data has to be filtered and the average velocity has to be calculated in a reasonable sampling period. In Germany, the averaging occurred on the entire sampling period where $15-20 \mathrm{~min}$ were collected from all the three ADCPs with sampling frequency of $1 \mathrm{~Hz}$. In Albania, the samples were shorter, but yet above $3 \mathrm{~min}$. If the opposite flow direction is denoted as a negative velocity, then the apparent velocity filtering follows the following simple conditions:

$$
\left\{\begin{array}{l}
v_{a f i}=N a N \text { if } v_{a i}<0 \\
v_{a f i}=N a N \text { if } v_{a i}>\bar{v}_{a i}+3 \frac{1}{N_{r a w}-1} \sum_{i}\left(v_{a i}-\overline{v_{a i}}\right)^{2}
\end{array}\right.
$$

where the $\boldsymbol{v}_{\boldsymbol{a}}$ is the raw apparent velocity and $\boldsymbol{v}_{\boldsymbol{a}}$ is the filtered apparent velocity. $i$ stands for the number of the ADCP sample. $N_{\text {raw }}$ is the total number of the raw data. The application of this simple filtering is mostly due to the known acoustic artefacts like aliasing, roughness frequency change, grazing angle variation, and high natural variability of the sediment transport causing decorrelation in the Doppler velocity calculation. Finally, to diminish the white noise, simple averaging of the filtered data is applied, considering only the filtered data. This filtering procedure is expected to deliver corrected magnitude and direction of the spatially averaged velocity of the bed. The spatially averaged velocity assumes not only the beam homogeneity but also the fact that each beam is highly influenced by the immobile river rough surface that can change from position to position. The highly dilute active layer 
(with porosity of $0.8-0.95$ ) allows the signal to penetrate to the immobile surface for any frequency.

\subsection{Physical samples data analysis}

The physical samples were analyzed and the transport rates were calculated together with the PSD specific for each sample. No correction of the transport rates ( Fig.2 plot b.) was applied, even though the study and the report of the BfG (German Institute of Hydrology, responsible for all measurements in Germany) clearly suggests that the average samples have to be corrected [8]. The potential loss of sandy part of the bed load was analyzed and tested under laboratory conditions in a flume resulting with 1.5-3.5 times less collecting mass than the real transport, due to the issues mentioned above [8]. This order of magnitude was also confirmed by other investigations [10]. The transport rates were compared with the apparent velocities and the ADCPs calculated transport rate (e.g. using the kinematic transport model). All three samples per position are directly included in the analysis, without averaging. The data from Albania was selectively chosen, where some unreasonable samples were discarded and the average of the available samples per position was correlated with the ADCPs.

\subsection{Kinematic Transport Model}

The riverbed surface explicitly incorporates the footprint of the immobile and mobile particles. Where the apparent velocity $\left(\boldsymbol{v}_{\boldsymbol{a}}\right)$ is assumed to be spatially averaged, equal to:

$$
v_{a f}=\frac{A_{i}}{A} v_{p}
$$

where $v_{p}$ is the average particle velocity and $A$ is the total sampling area, $A_{i}$ is the insonified area of the mobile particles. Rennie et al. [1] pointed out that the transport rate is equal to the product of the average particle velocity and the mass of the particle per unit area. Later, they extend the concept to the kinematic transport model:

$$
q_{s}=\sum_{i} v_{p_{i}}\left[\delta(1-\lambda) \frac{A_{i}}{A} \rho_{s}\right],
$$

where $q_{s}$ is the transport rate, $\delta$ and $\lambda$ are the thickness and the porosity of the active layer and $\rho_{s}$ is the density of the sediment (usually quartz $2.65 \mathrm{~g} / \mathrm{cm}^{3}$ ). It remains unclear how exactly the frequency and the sampling area/volume are influencing the $A_{i}$, but it is clear that each frequency penetrates differently trough the active layer and that bottom roughness significantly influences the instantaneous values of the apparent velocity. The filtering method is reducing this uncertainty and it makes the apparent velocity directly applicable in the transport model. However, there is considerable uncertainty of the highly dynamic active layer thickness and porosity. In this study, the variation of active layer thickness is considered as the main model uncertainty varying between two and eight times the median particle diameter $(D 50)$. The porosity is calculated according to the VanRijn equations [11] for bedload concentration, where $c_{b}=(1-\lambda)$. The shear velocity used in the VanRijn equations was calculated by using the assumption for log law profile in the lower part of the water velocities column obtained from the ADCPs (typically). It is also known that the log profile tends to fail if the data is not close enough to the boundary layer. It also may not perform good if the ADCP velocities are not processed properly or if the cell resolution does not fit the assumptions for the log-law region. In these cases, the shear velocity is calculated using the Kalugelan formula for shear velocity for rough boundaries that uses the depth-average velocity as an input. The active layer is expected to be much more diluted than the dune and riverbed porosity usually reported in the literature (e.g. higher than 0.35-0.4) [11]. 


\section{Results and discussion}

\subsection{The filtered apparent velocity}

The filtering of the BT velocity or the apparent velocity increased the values from all the frequencies (Fig. 2, (a.)) and produced vector direction that is more reasonable. The direction improvement is more obvious especially if you deal with shorter time series and the temporal averaging is not sufficient to smooth the data, such as the case of Albania (Fig.2. rhombs, markers). The filtering also deals with the fudge effect of the reattachment dune region where the high turbulent flow confuses the ADCP signal and produces unreasonable data (e.g. Oder River Germany).

The 3Mhz could be partially sensitive to the "water bias" if some highly concentrated suspended clouds of sediments pass through the sample volume and produce some spikes of very high velocities. These outliers were also discarded with the filtering procedure.

The variation of the bedload velocities is mostly due to the fact that the PSD changed from position to position, especially in the data from Albania (Fig 2. (a.), rhomb markers) and due to the different sampling positions associated with a different water depth and velocity. Therefore, the filtering is much heviewr in the Albania data where $D 50$ varies from $0.7 \mathrm{~mm}$ to $13.5 \mathrm{~mm}$. Additionally the very low transport rates are obviously due to the improper positioning of the sampler, clogging, blocked nozzle or human error, that is hard to be noted during the sampling but could be discarded later after the results from the ADCPs are processed and demonstrate some bedload velocity.
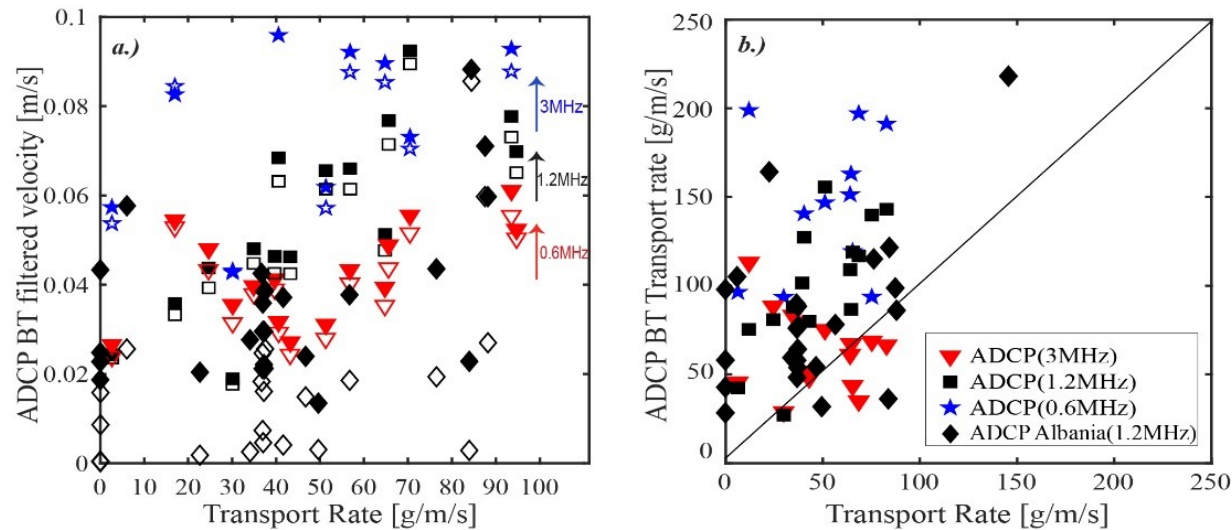

Fig. 2. (a.) Filtered (filled markers) and unfiltered (empty markers) apparent ADCP velocity demonstrating correlation with the physical transport rates; $(b$.$) Transport rates from the physical$ sampling on the $\mathrm{x}$-axis and the ADCP kinematic model on the $\mathrm{y}$-axis

Even after filtering and post-processing the data, there is difference between the velocities measured by ADCPs with different frequencies. Namely, the lowest frequency clearly demonstrates the lowest values especially in the area of higher transport rates (Fig. 2 (a.)). M9 SonTek delivered the highest apparent velocities working with $3 \mathrm{MHz}$. There are several factors that causes this effect but the most obvious one is that the lower frequency penetrates the active layer or deeper in the loose immobile bed and the received echo contains more data from the immobile surface than the active layer. The lower velocity is related with the Doppler shift calculation, thus with the echo identification in the range of the lowest part of the active layer where the immobile surface echoes may dominate. This is also related with the internal algorithm of the instrument that identifies the echoes used for calculating the BT velocity. Usually the algorithm considers the maximum echo of the small searching range. Considering that, the $0.6 \mathrm{MHz}$ have longer pulses and less acoustic sensitivity towards sand (as it is the dominant material in both campaigns), it is logical that the signal from the lower frequency would have larger input of the immobile surface bottom. On the other hand, the higher frequency waves contains more information from the mobile particle, hence they are 
dissipated in the active layer. It has to be noted that the mobile bed load layer has high porosity comparing to the immobile riverbed below, therefore any plane acoustic wave will partially contact the immobile surface. The more abounded transport the less the signal is biased by the immobile particles or surface, meaning less filtered data. The percentage of filtered data is increasing as the transport rate decreases. Finally, it has to be mentioned that the grazing angle of the emitted wave or the slanting angle of the beams in combination with the riverbed inclination and the inundations may contribute to different echo intensity and inaccurate echo identification by the internal algorithm.

\subsection{The transport rates}

The transport rates are calculated using the different frequencies, filtered apparent velocities and the kinematic model from eq. (3). The ADCP calculated transport rates are directly correlated with the transport rates obtained from direct physical measurements. Assuming that the filtering procedure filtered most of uncertainty in the data the variation of the transport rates is associated with the thickness of the active layer ( $\delta$ in eq. 4 . The data from Albania results with higher uncertainty due to the wider PSD and larger D50 of the samples and, thus the sensitivity of the active layer assumption. The values of the ADCP calculated transport rates vary with the ADCP frequency and the Van Rijn calculated concentration of the bedload $\left(c_{b}=1-\lambda\right)$ and the thickness is $5 D 50$ for Oder data and $3 D 50$ for Tommorice data. The thickness of the active layer is intentionally chosen with smaller variation due to the larger D50 and higher sorting coefficient of the PSDs in Albania. As it was mentioned before, the highest frequency with its filtered apparent velocity offers the highest transport rates boundaries. The non-corrected transport rates directly measured by the custom-made samplers are expected to underestimate the average bedload transport for sand in accordance with Hillebrand, et al and McLean at al [8, 12].

However, this makes more reasonable the values from the ADCPs, especially the ones with $1.2 \mathrm{MHz}$ and $3 \mathrm{MHz}$, complying with the assumptions. Fig 2 (b.) shows the uncertainty of th physical sample given by the horizontal bars. It should be noted that the std is out of the three value mean, no excluding eventual erroneous data such as zero values or bad positioning recorded on the videos. The videos from the mounted camera also showed that it could actually dig inside the dune and collect only that material instead of real transport occurring while the dune is migrating. They were also analyzed using velocity estimation algorithms (e.g. Optical Flow). This image analysis suggests that the bedload material was not properly entering the sampler due to the disturbed hydraulic conditions induced by the clogging of the smaller mesh $(0.5 \mathrm{~mm})$ in Germany.

The kinematic model data obviously overestimates some of the results, but in general, there is a good matching of the data. The deviation of the physical samplers is not presented because the number of samples per position was not consistent. The obviously too low transport rates in some measurements by the sampler are clearly part of the high physical sampling uncertainty. These data can be excluded from the total estimation of the transport rates and the ADCPs could easily identify these outliers and later to exclude them from the final average of the physical sample rates. One of the drawbacks of this model is the arbitrary chosen constant thickness of the active layer that in reality can vary in the mentioned constraints. Nevertheless, using two frequencies gives the opportunity of estimating the BsEI from the two frequencies and analyze the dependence of the $S V$ and $T S$ and the active layer thickness, hence the penetration changes as the thickness increases or a change of the PSD is present. This is an ongoing laboratory study and will be presented in the future work of the authors. Currently the chosen mean values of the thickness are related with the intensity of the transport and variation of the $B S E I$. In the supplementary material the deviation of each position is presented as a possible range $(2 D 50-8 D 50)$ of the ADCP measured transport rate. In the end, one can easily draw conclusion that even with the considered uncertainty of the ADCP values the transport rates are slightly higher than physical transport rates. This leads 
to the fact that the physical sampling has its disadvantages (seen on the recorded videos and laboratory tests) and a correction factor has to be chosen for a proper presentation.

\subsection{Conclusions}

The scarce number of measurements demonstrated that the ADCPs BT velocity can be used for relatively accurate estimation of the bedload transport rate. The filtering procedure decreased the uncertainty of the kinematic model and delivered its application without explicitly fitting parameters. In addition, they could help for a qualitative validation of the physical samples or support the determination of a correction factor if they are conducted together. Excluding the outliers improves the statistical validity of the physical sampling. The ADCP data gives a more comprehensive description of the erosion deposition process in the rivers and can be reported in a shorter periods (longer than 3 min averages). Additionally, with a-priori defined PSD of the bed load the kinematic model reported relatively good estimation of the bedload transport rates not just in comparison with the physical samples but also in compliance with the uncertainty of the bedload measurements. Attention should be paid on the frequency used and the predefined hydraulic conditions for estimating the active layer porosity and thickness. These two parameters contributes for the highest uncertainty of the kinematic model. The ADCP parameters settings are also very important in order to have reasonable resolution of the water velocity data. It remains unknown how the internal processing of the signal and the identification of the echoes influence the Doppler velocity calculation; this is only known by the manufacturer of the respective ADCP but not by the client/operator. The future work focuses on identifying the different types of bottom scattering and associating the active layer properties with the acoustic parameters. Additionally, all the sources of Doppler uncertainty should be identified and the procedure of filtering has to be improved and eventual in situ software should be developed for direct evaluation.

\section{References}

1. C. Rennie, R. G. Millar and M. A., ,. Church, "Measurement of bed load velocity using an acoustic Doppler current profile," Journal of Hydraulic Engineering, vol. 128(5), p. 473-483, 2002.

2. C. Rennie and P. V. Villard, "Site specificity of bed load measurement using an acoustic Doppler current profiler," J. Geophys. Res., vol. 109, no. F03003, 2004.

3. D. Gaeuman and R. B. Jacobson, "Acoustic bed velocity and bed load dynamics in a large sand bed river," $J$ Geophys Res Earth Surf, vol. 111(F2), no. F02005, 2006.

4. F. G. Latosinski, R. N. zupiany, M. Guerrero, M. L. Amsler and C. Vionnet, "The ADCP's bottom track capability for bedload prediction: Evidence on method reliability from sandy river applications," Flow Measurement and Instrumentation, vol. 54, no. June 2016, pp. 124-135, 2017.

5. C. Rennie, D. Vericat, R. Williams, J. Brasington and M. Hicks, Calibration of aDcp apparent bedload velocity to bedload transport rate, Tsutsumi, D., and Laronne, J., Wiley., 2017

6. S. Conevski, M. Guerrero, N. Ruther and C. Rennie, "ADCP performance of measuring the bedload velocity under changing conditions of particles mobility/bedload transport conditions," in submition, 2018.

7. R. I. R. Lee Gordon, “Acoustic Doppler Current Profiler Principles of Operation A Practical Primer," RD Instruments, San Diego, California, 1996.

8. G. Hillebrand and R. Frings, "Von der Quelle zur Mündung: Die Sedimentbilanz des Rheins im Zeitraum 1991 - 2010.," Internationale Kommission für die Hydrologie des Rheingebietes, Lelystad, Achen, 2017.

9. R. M. Frings and V. Stefan, "Guidelines for sampling bedload transport with minimum uncertainty," Sedimentology, 2017.

10. D. McLean, M. Church and B. and Tassone, "Sediment transport along the lower Fraser River. 1. Measurements and hydraulic computations," Water Resour. Res, vol. 35, pp. 2533-2548, 1999.

11. L. Van Rijn, "Sediment transport, part i: bed load transport," J. Hydraul. Eng., vol. 110, p. 1431-1456, 1984.

12. D. McLean, M. Church and B. Tassone, "Sediment transport along the lower Fraser River. 1. Measurements and hydraulic computations," Water Resour. Res., no. 35, pp. 2533-2548, 1999. 